\title{
Review
}

\section{Programmed cell death and patterning in Drosophila}

\author{
JC Rusconi ${ }^{1,2}$, R Hays ${ }^{1,2}$ and RL Cagan ${ }^{\star, 1}$ \\ 1 Department of Molecular Biology and Pharmacology, Washington University \\ School of Medicine, St. Louis, Missouri, MO 63110, USA \\ 2 The first two authors contributed equally to this review \\ * Corresponding author: RL Cagan, Department of Molecular Biology and \\ Pharmacology, Washington Univesity School of Medicine, Campus Box 8103; \\ 660 S. Euclid Avenue, St. Louis, Missouri 63110, USA. Tel: 314-362-7796 \\ Fax: 314-362-7058; E-mail: cagan@molecool.wustl.edu
}

Received 20.6.00; revised 14.8.00; accepted 23.8.00

Edited by S Kumar

\begin{abstract}
Selective cell death provides developing tissues with the means to precisely sculpt emerging structures. By imposing patterned cell death across a tissue, boundaries can be created and tightened. As such, programmed cell death is becoming recognized as a major mechanism for patterning of a variety of complex structures. Typically, cell types are initially organized into a fairly loose pattern; selective death then removes cells between pattern elements to create correct structures. In this review, we examine the role of selective cell death across the course of Drosophila development, including the tightening of embryonic segmental boundaries, head maturation, refining adult structures such as the eye and the wing, and the ability of cell death to correct for pattern defects introduced by gene mutation. We also review what is currently known of the relationship between signals at the cell surface that are responsible for tissue patterning and the basal cell death machinery, an issue that remains poorly understood. Cell Death and Differentiation (2000) 7, 1063-1070.
\end{abstract}

Keywords: Drosophila; apoptosis; patterning

Abbreviations: Dpp, decapentaplegic; EGFR, epidermal growth factor receptor; IAP, inhibitor of apoptosis protein; IrreC-rst, irregular chiasm C-roughest; JNK, Jun N-terminal kinase; PCD, programmed cell death; P/D, proximo-distal; Tkv, thick veins; $\mathrm{Wg}$, Wingless

\section{Introduction}

The selective removal of cells by programmed cell death (PCD) is a common and fundamental aspect of developmental maturation. For example, perhaps half of the neurons created in the developing central nervous system are subsequently removed by PCD. In $C$. elegans, programmed cell death is used to remove more than $10 \%$ of the cells initially created, and in Drosophila PCD is used extensively to refine developing structures.
Why create cells only to later remove them? Three explanations have been proposed for this seeming paradox. The first is an evolutionary argument, in which current structures are refined examples of larger ancestral ones, and the simplest structural modification is removal of unnecessary cells. Related to this is the use of cell divisions to segregate neuronal determinants to one daughter and subsequent removal of its sister. Second, neurons have been demonstrated to 'compete' for growth factors to create optimal synaptic connections; this competition permits coordination between neurons during the formation and refinement of neural pathways. ${ }^{1}$ Third, PCD provides an important tool during development to permit epithelial patterning without extensive use of lineal information. In this review, we examine the role of PCD in patterning, a central aspect of animal maturation.

The term 'patterning' can have a variety of meanings. We define patterning as a reiterative process that imposes spatial organization onto developing tissues. The complexity of tissues in multicellular organisms necessitates the use of patterning mechanisms. By propagating patterned spatial cues across a developmental field, a relatively small number of signals can be used to organize structures such as early segmental boundaries, the developing nervous system, and the developing retina. Overlaying multiple patterned signals can then create complexity. As described below, the best understood role for PCD during the patterning process is to help sculpt tissue and to provide spatial precision.

\section{Segmentation}

One important use of PCD is during the process of segmentation. For example, in the mammalian hindbrain, segmental boundaries are established in part by removal of misplaced cells between rhombomere borders. ${ }^{2}$ An apparently similar process can be seen during segmentation of the Drosophila embryo. The Drosophila embryo is divided into 14 segments along its anterior/posterior axis. Using time lapse video microscopy and vital markers to visualize segmental position and cell death, Pazdera et $a l^{3}{ }^{3}$ created a map of cell death in the developing segments of living embryos. They found that, midway through embryogenesis (stages 12-14), three-quarters of the dying cells are in or adjacent to the segment border. ${ }^{4}$ This suggests a role for cell death in refining this border. The dying cells appear to form three clusters along the dorsal/ventral axis (Figure 1). Interestingly, expression of the death effector reaper occurs at these stages in similar clusters along the segmental border.

What regulates this cell death? The identity and size of each segment is set by a cascade of regulatory genes including the 'segment polarity genes' that provide the cells within each segment with their positional identity. Previous 
studies demonstrated that embryos mutant for the segment polarity genes are shorter and have increased PCD, suggesting that at least some miss-specified cells are removed. ${ }^{2,3}$ For example, reducing the activity of wingless or its downstream target armadillo resulted in a fivefold increase in the number of dying cells. ${ }^{3}$ This ectopic death was found exclusively within the anterior compartment of each developing segment, the region in which wingless signaling is active. In contrast, embryos mutant for the posterior identity gene naked exhibited a sixfold increase in cell death specifically in the posterior compartment of each segment.

A simple interpretation of these results suggests that wingless signaling promotes survival in the anterior region of each segment while naked activity promotes survival in posterior regions. However, embryos mutant for both wingless and naked are larger than embryos mutant for wingless alone, suggesting a more complicated mechanism for cell death signaling in the segments. ${ }^{4}$ Perhaps, similar to mammalian rhombomere development, cells at the border influence the survival of their neighbors across the segmental boundary, removing inappropriately-placed or
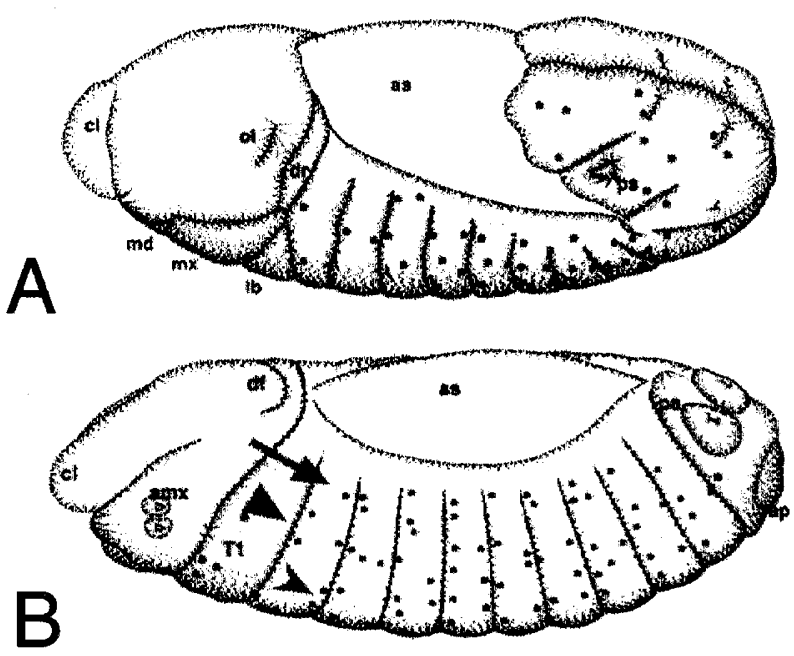

Figure 1 PCD fate map of wild-type embryos at stage $12(\mathbf{A})$ and $13(\mathbf{B})$ of embryogenesis. The three clusters of $A O$ positive cells at the segment border are marked with an arrow (dorsal cluster), arrowhead (mid-lateral cluster), and a concave arrowhead (ventrolateral cluster), taken from Pazdera et al. ${ }^{3}$ ectopic cells. This mechanism would help ensure precisely defined boundaries.

\section{Midline glia}

Another segmented structure that makes use of PCD during its development is the ventral midline, a portion of the central nervous system roughly analogous to the mammalian spinal cord. ${ }^{5}$ The ventral midline arises from the embryonic mesectoderm, a two cell-wide stripe that extends the length of the embryo and forms a boundary between the ectoderm and mesoderm. Cells within this region proliferate, differentiate, and in some cases die, producing three types of glia (MGA, MGM and MGP) and three types of neurons (VUM, MP1 and UMI). ${ }^{6-10}$ These cells will eventually form the basis of the commissural axon tracts. $^{7}$ The simplicity of the ventral midline has made it an increasingly popular system for examining the role of cell death in embryonic differentiation.

At the end of embryogenesis (stage 17), CNS midline cells are located in pockets separated by basement membrane pores that extend the length of the nerve cord (Figure 2). ${ }^{11}$ During normal development, embryos lose $50 \%$ of their initial glial cells by the end of embryogenesis. $^{12}$ In the presence of a deficiency (H99) that blocks PCD, the pores accumulate extra cells due to a 3-5-fold increase in midline glial cell numbers. Interestingly, these ectopic cells retain many properties of midline glia. They project toward and appear to contact the axon commissures and even ensheath neurons. ${ }^{11,12}$ Not surprisingly, these extra midline glial cells disrupt the CNS midline. The axon scaffolding is normal in $H 99$ mutants but there is thickening at the junctions between the commissural and longitudinal axon tracks and the nerve cord fails to condense. $^{11}$

The numbers of midline neurons was not affected in $\mathrm{H} 99$ mutants and they do not appear to undergo PCD in wildtype embryos. ${ }^{11,12}$ Instead, neurons play a different role regarding PCD at the midline. In commissureless mutant embryos the commissural axons fail to cross the ventral nerve cord, resulting in very little midline glia-axon contact. The stage of maximal cell death in these embryos is shifted to an earlier embryonic stage and the overall death of the MGA and MGM glia increases twofold. ${ }^{12}$ This suggests that contact by axons plays an important role in regulating the death of glia.
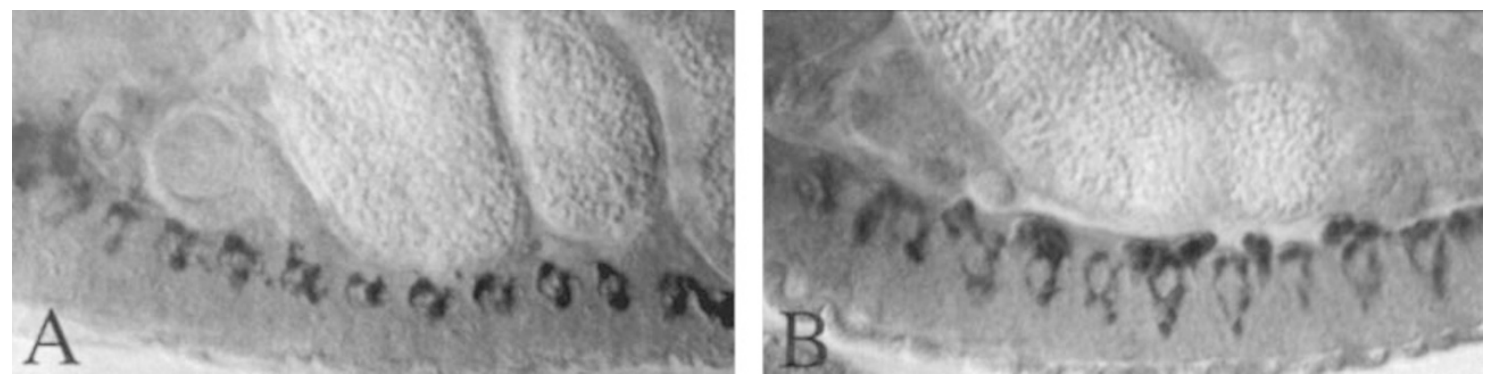

Figure 2 H99 mutant embryos have extra midline cells. Anti-B galactosidase expression labeling midline glia (P[4.5 slit/lacZ]) in wild-type (A) and H99 (B) mutant embryos. Taken from Zhou et al. ${ }^{11}$ 
What is the molecular basis of this regulated death at the ventral midline? Little is understood of the signals that trigger death in some glial cells but spare others. Downstream, death occurs by apoptosis and can be blocked by the caspase inhibitor P35. ${ }^{13}$ Also involved are the caspase effectors reaper, grim, and hid, which are expressed in subsets of cells in the midline and appear to act cooperatively to remove excess cells. ${ }^{11}$

At least two cell surface signaling pathways mediate cell death in the ventral midline. Mutations that affect dEGFR activity alter patterns of midline cell death. Gain-of-function dEGFR embryos contain ectopic midline glial cells, whereas loss of function dEGFR embryos have no midline glia at all. ${ }^{14}$ In many tissues, dEGFR pathway activity is controlled by the transmembrane effector Rhomboid, and the midline is no exception. Rhomboid is expressed in all midline cells by mid-embryogenesis (stage 11). ${ }^{15}$ Rhomboid also inhibits cell death at the midline: embryos lacking rhomboid activity retain one (vs three) midline glial cell per segment, and ectopic expression of Rhomboid leads to an initial 50\% increase in MGA and MGM glial (remarkably, cell numbers recover by the end of embryogenesis). ${ }^{15,16}$ In contrast, mutations in the secreted dEGFR inhibitor Argos lead to an increase in midline glial cell numbers. ${ }^{9}$ Together, these data indicate that $d E G F R$ signaling is required to promote 'life' in the embryonic midline glia; a similar role in the developing retina is discussed below.

Less clear is the role of the Notch signal transduction pathway, which also regulates cell number in the embryonic midline. A reduction in Notch signaling leads to a loss of midline cells. ${ }^{10,16,17}$ This reduction is due at least in part to a change in cell fates at the midline; whether Notch signaling can also directly affect cell death in the midline has yet to be assessed.

\section{Head development}

When major changes occur in the shape or structure of a tissue, PCD is suspected of playing a role. This type of death has been coined morphogenetic cell death. During embryonic development, a number of major events reshape and remodel the head. ${ }^{18}$ Many of these morphogenetic movements involve cell death. White et al. demonstrated that reaper mRNA was expressed in a number of dying cells in the developing embryonic head region. ${ }^{19}$ Although not every cell that expresses reaper will actually die, expression of reaper appears to convey a high potential for PCD suggesting that the developing head makes great use of death during its morphogenesis. Indeed, all morphogenetic cell movements in the head are accompanied by extensive reaper expression including: reduction in size of most of the head epidermis, intercalation in the lateral movement of the optic lobe primordia, delamination in the dorsomedial brain, invagination in the optic lobe, involution in the dorsal pouch, separation of epithelia in the optic lobe after invagination, and fusion of the antennal and maxillary lobes. ${ }^{20}$

Surprisingly, in the absence of PCD (in H99 mutant embryos) some of these processes still occurred normally, particularly early events. ${ }^{20}$ PCD is clearly used extensively in these early morphogenetic movements; apparently, the need for PCD is overcome in $\mathrm{H} 99$ mutants by other mechanisms such as cell size reduction. Interestingly, Nassif et al. ${ }^{20}$ note that those early morphogenetic events that can occur in the absence of PCD appear to be more conserved phylogenetically. This suggests that PCD may have evolved more recently as a regulatory mechanism for morphogenesis.

\section{Reorganization of tissues during metamorphosis}

During insect metamorphosis, larval tissues undergo dramatic reorganization to establish the adult body plan. While much of this reorganization involves the proliferation and outgrowth of larval tissues (e.g. appendages), many larval tissues are eliminated through PCD during or shortly after metamorphosis. In Drosophila, levels of the steroid hormone ecdysone control the programmed death of many such tissues including the larval salivary gland and midgut and the post-metamorphic degeneration of specific populations of muscle and central nervous system cells. Both high and low levels of ecdysone can initiate PCD in a tissue-specific fashion.

Like their embryonic progenitors, the larval midline glia are thought to play a role in stabilizing outgrowing neurites that run along or cross the midline. As such, they serve as an important template during patterning of the CNS. Following the completion of neural pathfinding, the midline glia are no longer required and roughly half undergo apoptosis late in embryogenesis. ${ }^{12}$ The remaining cells survive and proliferate during subsequent larval development, ${ }^{12,21-24}$ but are also eliminated by $80 \%$ completion of metamorphosis. ${ }^{25}$ Tissue culture experiments suggest that the peak of ecdysteroid production that occurs at puparium formation signals the cessation of cell proliferation and the onset of death. ${ }^{25}$

Histolysis of the larval midgut and salivary gland are also mediated by high levels of ecdysone, although at different developmental stages. ${ }^{26}$ The larval midgut contains polyploid epithelial cells that lie on an external basement membrane. Islands of diploid cells surrounding the basement membrane proliferate during early pupal development and surround these larval cells to form the adult midgut; the remains of the larval structure are discharged as the meconium soon after enclosure. ${ }^{27,28}$ Degeneration of the salivary gland occurs very abruptly and is complete by $15 \mathrm{~h}$ after puparium formation. Diploid cells anterior to the gland proliferate to form the adult tissue during pupal development. $^{28}$

The elimination of these structures is stereotypically apoptotic. Degenerating cells show nuclear staining by acridine orange and DNA fragmentation, and can be rescued by expression of the caspase inhibitor $p 35 .^{26}$ In addition, expression of the death effector genes reaper and hid are up-regulated in both of these tissues just prior to the onset of death, while expression of the cell death inhibitor diap2 is repressed. ${ }^{26}$ These studies suggest that PCD is both positively and negatively regulated in these tissues, and that ecdysone levels direct larval tissue reorganization through stage-specific, spatially restricted effects on gene expression. 


\section{The emerging adult}

Newly emerged adults also demonstrate extensive cell death in both nervous and muscle tissue. For example, many of the abdominal muscles required for eclosion and wing spreading undergo apoptosis within the first $12 \mathrm{~h}$ of adulthood, and the associated neurons die within the next $12 \mathrm{~h}$ period. ${ }^{29-31}$ Little is known about the signals that direct muscle degeneration. Ligation experiments suggest that the signals originate in an anterior region of the fly, and the timing of the signal implicates eclosion hormone. ${ }^{32}$ The signal for muscle degeneration is clearly initiated before eclosion begins, and cell death can be suppressed by delaying adult eclosion through enforced ecdysis. ${ }^{32}$ Characterization of the $m c d-1$ and $m c d-2$ (muscle cell death) mutations which delay cell death in a subset of adult muscle groups ${ }^{33}$ may provide information about the general mechanism for these death decisions.

Neural death in the adult is initiated after the adult fly emerges from the pupal case and is clearly regulated by ecdysone levels. ${ }^{32,34}$ Approximately 300 neurons in the ventral nerve cord undergo PCD. These cells, referred to as type II neurons, selectively express the $A$ isoform of the ecdysone receptor (EcR-A) at 10-fold higher levels than neurons not fated to die. ${ }^{34-37}$ EcR-A expression is upregulated early in metamorphosis and persists throughout pupal development. Apoptosis in these neurons is triggered by declining ecdysone levels at the end of metamorphosis and can be delayed by treatment with the ecdysone analog 20-hydroxysterone at least $3 \mathrm{~h}$ prior to the onset of degeneration. ${ }^{34}$ This suggests that these neurons achieve a competence to die and that ecdysone activity inhibits the initiation of cell death. Consistent with this view, reaper and grim transcripts accumulate prior to PCD in the same subset of ventral neurons that express high levels of EcR-A. ${ }^{36}$ Similar to postmetamorphic muscle degeneration, the death of type II neurons is mediated by a signal from the anterior of the animal as well as by ecdysone levels; both signals are required for the accumulation of reaper transcripts. ${ }^{36}$ The cell death signal emanating from the anterior of Drosophila has not been identified. It may be homologous to the neurocidin protein of the moth Manduca sexta, which acts to selectively kill MN12 neurons after eclosion. $^{38}$

\section{Sculpting of adult tissues - the retina}

The Drosophila compound eye is composed of approximately 750 individual unit eyes, or ommatidia, precisely positioned within a highly ordered crystalline lattice (Figure 3). Each ommatidium is an assembly of 14 cells: eight photoreceptors, four cone cells, and two primary pigment cells. Elaboration of the ommatidial lattice begins late in larval development when ommatidial precursor clusters emerge in a wave of patterned differentiation. ${ }^{39}$ As the morphogenetic furrow passes, cells arrest in $\mathrm{G} 1$ of the cell cycle and the ommatidial lattice begins to form with the differentiation of the first photoreceptor neuron, R8. Additional cells are sequentially recruited to the growing ommatidial cluster through signals emanating from previously differentiated cells, culminating in the 14-cell group by early pupal development. Ultimately, individual ommatidia are positioned within a hexagonal lattice of shared secondary and tertiary $\left(2^{\circ} / 3^{\circ}\right)$ pigment cells (Figure 3 ).

Assembly of the pigment cell lattice is absolutely dependent on spatially restricted programmed cell death. By $24 \mathrm{~h}$ of pupal development $\left(25^{\circ} \mathrm{C}\right)$ all 14 ommatidial cells have been selected, leaving a large number of undifferentiated cells in the interommatidial space. Approximately one-third of these cells are eliminated through PCD between 24 and $40 \mathrm{~h}$ of pupal development in order to refine the pigment cell lattice. In general, interommatidial cells making contact with primary pigment cells are selected to survive, while those removed from contact with the primaries are fated to die. ${ }^{40,41}$

The mechanism by which cells are directed to undergo PCD involves a complex interplay between signaling by the cell surface receptors Notch and dEGFR (DER). Interference with Notch signaling blocks formation of the primary pigment cells and also cell death, ${ }^{42,43}$ suggesting a role for these cells in the regulation of PCD. Laser ablation studies support this view. Direct ablation of the cone and primary pigment cells results in the death of neighboring interommatidial cells; in contrast, ablation of the photoreceptor
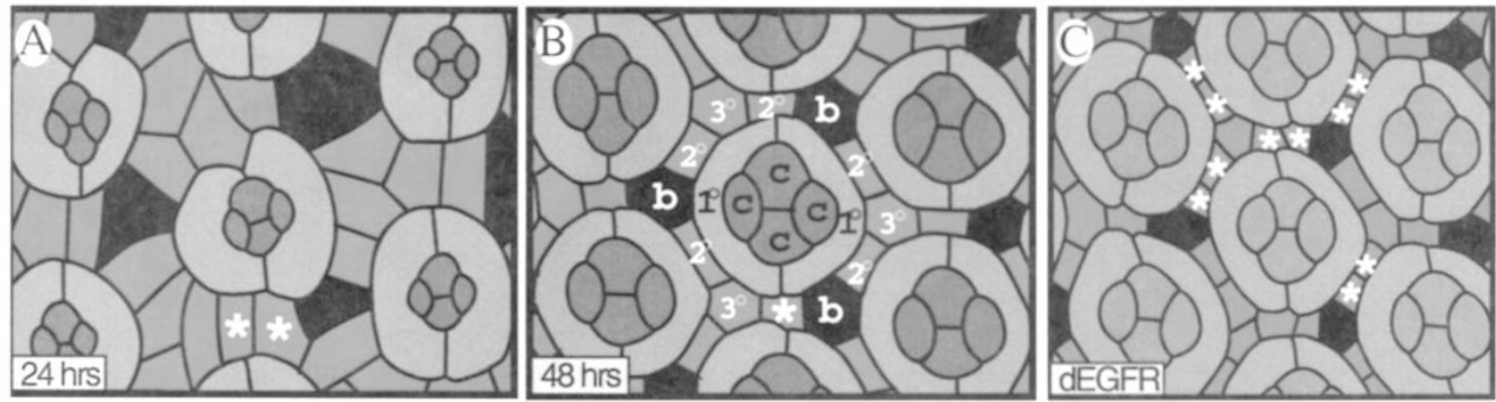

Figure 3 Tracing of the surface of a pupal retina before $(\mathbf{A})$ and after $(\mathbf{B}, \mathbf{C})$ the stage of programmed cell death. (A) In the young wild-type pupal retina, ommatidia are composed of cone cells and primary pigment cells; photoreceptor neurons are below the plane of this view and are not shown. Ommatidia are still separated by a variable number of $2^{\circ} / 3^{\circ}$ precursor cells. Two precursors in a single niche are denoted by asterisks $\left({ }^{*}\right)$. (B) In the more mature pupal retina, selective PCD has removed several precursor cells; the remaining $2^{\circ} / 3^{\circ} \mathrm{s}$ are reorganized into an interweaving hexagonal lattice. Note that a single $2^{\circ} / 3^{\circ}$ cell $\left({ }^{*}\right)$ is found in the same niche where previously multiple cells were found (see asterisks in panel $\mathbf{A}$ ). (C) Expression of an activated form of dEGFR leads to a block in PCD and excess $2 \%$ $3^{\circ} \mathrm{s}$; asterisks indicate examples of multiple cells in a niche where only a single $2^{\circ} / 3^{\circ}$ is normally found. Abbreviations: $c=c o n e$ cell; $1^{\circ}, 2^{\circ}, 3^{\circ}=$ primary, secondary, and tertiary pigment cell, respectively; $b=$ bristle 
neurons had no effect. ${ }^{44}$ This suggests that the cone cells and primary pigment cells provide a survival signal to neighboring $2 \% 3^{\circ}$ precursors. Direct ablation of the cone and primary pigment cells results in the death of neighboring interommatidial cells, however, and not a loss of death as seen in Notch mutants. ${ }^{44}$ Furthermore, mutations in Notch block cell death even when the cone and primary pigment cells are simultaneously ablated. ${ }^{44}$ This suggests a model in which Notch acts within the interommatidial lattice to promote cell death, and cells of the ommatidia send a life signal to immediately adjacent cells. Interestingly, reducing Notch function at other stages of retinal development had no effect on PCD, suggesting that the sensitivity of $2^{\circ} / 3^{\circ}$ precursor cells to death signaling by Notch is temporally regulated. ${ }^{42}$

One counterbalance to the Notch death signal is a rasmediated life signal originating within the ommatidium. dRAS-1 is known to be involved in a variety of cell fate decisions in the developing eye and opposes Notch signaling in pupal PCD. Expression of an activated $d R A S-1$ throughout the eye prior to the onset of death results in complete rescue of interommatidial cells whether or not cone and primary pigment cells are present. ${ }^{44}$ As described above, ablation of the cone and primary pigment cells results in increased death of interommatidial cells, identifying cells within the ommatidium as a source of the survival signal. Activation of the $d R A S-1$ pathway in this process is signaled through dEGFR. $d E G F R$ signaling is critical for cell fate specification in the eye, and loss-offunction mutations in $d E G F R$ result in excessive cell death in the interommatidial lattice. ${ }^{45-47}$ Expression of an activated $d E G F R$ throughout the pupal eye results in substantial rescue of cell death in these same cells, ${ }^{44}$ while widespread expression of argos, an antagonistic ligand of $d E G F R$, results in excessive PCD through inhibition of the $d R A S-1$ pathway. ${ }^{45,48} d R A S-1$ acts to promote cell survival by repressing the expression of head involution defective (hid), ${ }^{49,50}$ which induces PCD through activation of a caspase pathway. ${ }^{13,51}$

Study of the morphogenesis of the $2 \% 3^{\circ}$ pigment lattice maturation and the involvement of local signaling through Notch and $d E G F R$ suggests that specific cell-cell contacts are involved in the selection of cells to undergo apoptosis. Additional support for this view comes from mutational analysis of irregular chiasm $C$-roughest (irreC-rst). IrreC-rst encodes a transmembrane protein that demonstrates homophilic binding in tissue culture cells. ${ }^{52,53}$ It is expressed dynamically in most cell types during retinal development. Prior to the onset of cell death, IrreC-rst accumulates at the borders between primary pigment cells and interommatidial cells in a Notch-dependent manner. ${ }^{41,54}$ During this period, interommatidial cells reorganize into chains, lying end-to-end between ommatidia. Without this cell sorting and reorganization, apoptosis is blocked in the $2{ }^{\circ} / 3^{\circ}$ lattice and the hexagonal array of the adult eye is disrupted. ${ }^{41}$ Based on their retinal phenotype, mutations in the echinus locus appear to function downstream of irreC-rst in this process: $2^{\circ} / 3^{\circ}$ precursor cells align properly in echinus mutants, but apoptosis does not occur. $^{55}$ Mutations in echinus have no effect on the irre $C$ - rst mutant phenotype. ${ }^{41}$ The nature of the Echinus protein is unknown.

Together, these data suggest a general model in which PCD is promoted by Notch signaling within the interommatidial lattice cells and opposed by $d E G F R$ signaling initiated by the cone or primary pigment cells (Figure 4). Consistent with this model, Notch expression is limited to the interommatidial lattice cells during the period of pupal cell death, ${ }^{56}$ and $d E G F R$ expression is restricted to the same cells. ${ }^{44}$ spitz, which encodes a diffusible ligand of $d E G F R$, is expressed in the cone and primary pigment cells, and may represent the ommatidial life signal. ${ }^{44}$ IrreC-rst appears to function in regulating interommatidial cell organization, which is critical for lattice cell removal to proceed.

In addition to the lattice refinement described above, PCD occurs during two additional periods of eye development. At mid-pupal development $(60-70 \mathrm{~h})$, stunted ommatidia at the periphery of the retina are eliminated through apoptosis. ${ }^{55}$ These ommatidia, referred to as the perimeter clusters, are present at the end of nearly every ommatidial row. They frequently contain the full complement of photoreceptors, cone cells, and primary pigment cells, but they are reduced in size compared to internal ommatidia. The $2 \% 3^{\circ}$ pigment cells surrounding perimeter clusters survive development and serve to extend the interommatidial lattice to the edge of the retina. ${ }^{55}$

Low-level apoptosis also takes place earlier in development, in the larval eye imaginal disc. During the wave of

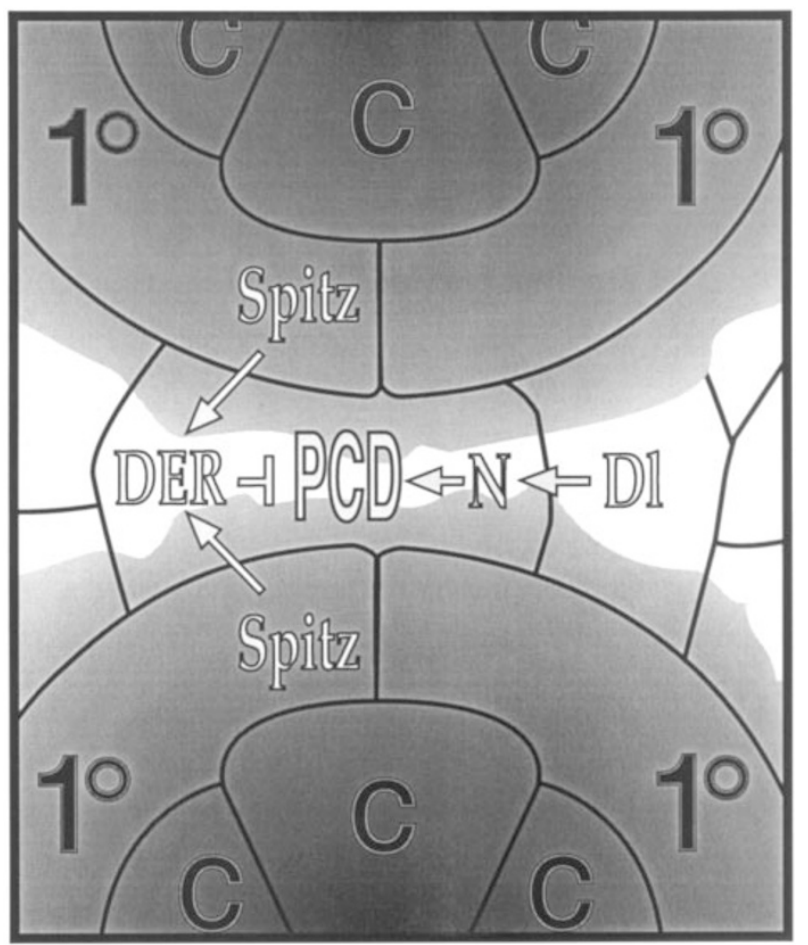

Figure 4 A model for patterning the $2 \% 3^{\circ}$ lattice. Spitz (gray) is secreted into the interommatidial space, rescuing $2^{\circ} / 3^{\circ}$ cells through activation of dEGFR Surviving cells then kill their neighbors by activating Notch. See text for details 
morphogenesis in the third instar eye disc, two significant bands of apoptotic cell death are visible: one at the posterior margin of the eye, and one just anterior to the morphogenetic furrow. ${ }^{55,57}$ The significance of this cell death is not known. It is possibly involved in regulating the number and quality of ommatidial precursor cells and the removal of cells that fail to achieve coordinated cell cycle arrest.

Because of the extreme regularity of pattern in the Drosophila retina, it is exceptionally sensitive to perturbation. Even minute changes in lattice patterning result in an adult rough eye phenotype. As such, a host of mutations have been identified that result in inappropriate cell death in the eye, many of them in genes not known to function overtly in retinal PCD. Mutations in genes required for specification of the eye field, morphogenetic furrow progression, cell division and differentiation, and neural maintenance all result in increased PCD in the retina (reviewed in ref. ${ }^{58}$ ). Recently, several new mutations that specifically affect cell death in the eye were identified in a genetic screen for enhancers and suppressers of the Irre Crst loss-of-function phenotype. ${ }^{59}$ Characterization of these loci will no doubt increase our understanding of this component of retinal patterning.

\section{Patterning repairs}

Several lines of evidence suggest that many cell types survive development through an active repression of apoptotic machinery. This repression is coupled to signal transduction cascades important for cell fate specification. If the balance of critical patterning signals becomes skewed, this repression is lost and apoptosis is initiated to remove cells that have lost proper positional information.

In the Drosophila wing imaginal disc, for example, the overlap of Wingless $(\mathrm{Wg})$ and Decapentaplegic (Dpp) in the presumptive wingblade is required for appendage outgrowth and patterning of the proximo-distal (P/D) wing axis. Recent data suggest that Dpp and Wg act together to downregulate activity of Jun N-terminal Kinase (JNK) in the wing. When the balance of $\mathrm{Wg} / \mathrm{Dpp}$ signaling is perturbed, $P / D$ positional identity is distorted and cells are subsequently removed through JNK-mediated apoptosis. ${ }^{1}$ In this fashion, normal metamorphosis can be quickly restored when cell fate specification goes awry. Similarly, the de novo synthesis of sphingolipids in the wing discs is also required to maintain downregulation of JNK signaling. Mutations that disrupt sphingolipid synthesis result in the JNK-mediated apoptosis of wing imaginal cells. ${ }^{60}$

Activation of JNK signaling may be a general mechanism for the initiation of PCD in Drosophila imaginal tissues, as has been suggested for vertebrates. ${ }^{61}$ It is well established that the eye imaginal disc undergoes extensive PCD during development (described above), and JNK signaling maybe involved its regulation. Overexpression of transforming growth factor $\beta$ activated kinase 1 (TAK1) in the eye disc leads to ectopic activation of JNK signaling and widespread PCD, ${ }^{62}$ suggesting that, as in the wing, both Dpp and JNK signaling may regulate cell death. However, the involvement of TAK1/JNK signaling in developmentally regulated cell death remains to be shown by mutational analysis.

Interestingly, Dpp has been implicated in the regulation of cell death in another capacity as well. The Drosophila inhibitor of apoptosis proteins (Diaps) interact with the Dpp type I receptor Thick veins (Tkv). Diap1 has been shown to bind Tkv in vitro, and both Diap1 and Diap2 associate with Tkv in vivo, ${ }^{63}$ suggesting that these proteins may function to inhibit apoptosis regulated by the Dpp signal transduction cascade.

Recent data suggests that PCD is a major mechanism for the recovery of positional information following disruptions in embryonic patterning. Namba et al. have manipulated the dosage of bicoid $(b c d)$ in order to observe how and to what extent embryonic pattern repair occurs. ${ }^{64}$ Animals with an altered bcd dosage develop compressions and expansions in the embryonic fate map that can be seen as abnormalities in segmentation gene expression. Below a certain threshold of disruption, these defects are repaired and the embryos developed into relatively normal larvae and adults. Strikingly, expansions in the fate map are repaired through cell death, but compressions are not repaired through cell proliferation. Rather, there is a concomitant decrease in cell death in compressed areas that, in combination with increased death in the expanded areas, restores the embryonic fate map. Within limits, the greater the extent of the patterning error, the greater the alterations in cell death.

\section{Final comments}

Studies in Drosophila have demonstrated a central patterning role for PCD during all stages of development. Indeed, PCD is used to selectively remove cells during sculpting of nearly every tissue examined. PCD is also commonly used for the removal of obsolete larval tissues, and is a highly effective mechanism for correction of early defects inherent in non-lineal based development. Perhaps the greatest challenge in understanding the connection between PCD and patterning is to understand how death signals are applied with spatial precision. That is, to understand the links between PCD and the specification of positional information during morphogenesis.

\section{References}

1. Adachi-Yamada T, Fujimura-Kamada K, Nishida Y and Matsumoto K (1999) Distortion of proximodistal information causes JNK-dependent apoptosis in Drosophila wing. Nature 8: 166-169

2. Graham A, Heyman I and Lumsden A (1993) Even-numbered rhombomeres control the apoptotic elimination of neural crest cells from odd-numbered rhombomeres in the chick hindbrain. Development 119: 233-245

3. Pazdera T, Janardhan P and Minden J (1998) Patterned epidermal cell death in wild-type and segment polarity mutant Drosophila embryos. Development 125 : $3427-3436$

4. Bejsovec A and Wieschaus E (1993) Segment polarity gene interactions modulate epidermal patterning in Drosophila embryos. Development 119:501 517

5. Crews S, Franks R, Hu S, Matthews B and Nambu JR (1992) Drosophila singleminded gene and the molecular genetics of CNS midline development. J. Exp. Zool. 261: $234-244$ 
6. Jacobs $\mathrm{J}$ and Goodman C (1989) Embryonic development of axon pathways in the Drosophila CNS I. A glial scaffold appears before the first growth cones. J. Neurosci. 9: 2402-2411

7. Klambt C, Jacobs J and Goodman C (1991) The midline of the Drosophila central nervous system: a model for the genetic analysis of cell fate, cell migration, and growth cone guidance. Cell 64: 801-815

8. Wharton Kand Crews S (1993) CNS midline enhancers of the Drosophila slitand Toll genes. Mech. Dev. 40: 141-154

9. Schweitzer R, Howes R, Smith R, Shilo B and Freeman M (1995) Inhibition of Drosophila EGF receptor activation by the secreted protein Argos. Nature 376: 699-702

10. Rusconi J and Corbin V (1999) A widespread and early requirement for a novel Notch function during Drosophila embryogenesis. Dev. Biol. 215: 388-398

11. Zhou L, Schnitzler A, Agapite J, Schwartz L, Steller H and Nambu J (1997) Cooperative functions of the reaper and head involution defective genes in the programmed cell death of Drosophilacentral nervous system midline cells. Proc. Natl. Acad. Sci. USA 94: 5131-5136

12. Sonnenfeld M and Jacobs R (1995) Apoptosis of the midline glia during Drosophila embryogenesis: a correlation with axon contact. Development 121: $569-578$

13. Hay BA, Wolff T and Rubin GM (1994) Expression of baculovirus P35 prevents cell death in Drosophila. Development 120: 2121-2129

14. Stemerdink $C$ and Jacobs R (1997) Argos and Spitz group genes function to regulate midline glial cell number in Drosophila embryos. Development 124: 3787-3796

15. Lanoue B and Jacobs R (1999) rhomboidfunction in the midline of the Drosophila CNS. Dev. Genet. 25: $321-330$

16. Menne T and Klambt C (1994) The formulation of commissures in the Drosophila CNS depends on midline cells and the Notch gene. Development 120: 123-133

17. Martin-Bermudo M, Carmena A and Jimenez F (1995) Neurogenic genes control gene expression at the transcriptional level in early neurogenesis and in mesectoderm specification. Development 121: 219-224

18. Campos-Ortega J and Hartenstein V (1997) The Embryonic Development of Drosophila melanogaster. 2nd edn. (Berlin: Springer-Verlag): 321-340

19. White K, Grether M, Abrams J, Young L, Farrell K and Steller H (1994) Genetic control of programmed cell death in Drosophila. Science 264: 677-683

20. Nassif C, Daniel A, Lengyel J and Hartenstein V (1998) The role of morphogenetic cell death during Drosophila embryonic head development. Dev. Biol. 197: 170-186

21. Truman JW and Bate CM (1988) Spatial and temporal patterns of neurogenesis in the CNS of Drosophila melanogaster. Dev. Biol. 125: 146-157

22. Taylor BJ and Truman JW (1992) Commitment of abdominal neuroblasts to a male or female fate is dependent on genes of hte sex-determining hierarchy. Development 114: 625-642

23. Prokop A and Technau G (1994) BrdU incorporation reveals DNA replication in non-dividing glial cells in the larval abdominal CNS of Drosophila. Roux's Arch. Dev. Biol. 204: 54-61

24. Bossing T and Technau G (1994) The fate of the CNS midline progenitors in Drosophila as revealed by a new method for single cell labeling. Development 120: $1895-1906$

25. Awad TA and Truman JW (1997) Postembryonic development of the midline glia in the CNS of Drosophila: proliferation, programmed cell death, and endocrine regulation. Dev. Biol. 187: 283-297

26. Jiang C, Baehrecke EH and Thummel CS (1997) Steroid regulated programmed cell death during Drosophila metamorphosis. Development 124: 4673-4683

27. Bodenstein D (1965) The postembryonic development of Drosophila. In Biology of Drosophila. Demerec M, ed (New York: Hafner Publishing Co.): 275-364

28. Robertson CW (1936) The metamorphosis of Drosophila melanogaster, including an accurately timed account of the principal morphological changes. J. Morph. 59: $351-399$

29. Finlayson LH (1975) Development and degeneration. In Insect Muscle. Unsherwood P, ed (New York: Academic)

30. Truman JW (1983) Programmed cell death in the nervous system of an adult insect. J. Comp. Neurol. 216: 445-452

31. Truman JW (1984) Cell death in invertebrate nervous systems. Annu. Rev. Neurosci. 7: 171-188

32. Kimura Kand Truman JW (1990) Postmetamorphic cell death in the nervous and muscular systems of Drosophila melanogaster. J. Neuroscience 10: 403-411
33. Kimura K and Tanimura T (1992) Mutants with delayed cell death of the ptilinal head muscles in Drosophila. J. Neurogenet. 8: 57-69

34. Robinow S, Talbot WS, Hogness DS and Truman JW (1993) Programmed cell death in the Drosophila CNS is ecdysone-regulated and coupled with a specific ecdysone receptor isoform. Development 119: 1251-1259

35. Fahrbach SE and Truman JW (1989) Autoradiographic identification of ecdysteroid-binding cells in the nervous system of the moth Manduca sexta. J. Neurobiol. 20: $681-702$

36. Robinow S, Draizen TA and Truman JW (1997) Genes that induce apoptosis: transcriptional regulation in identified doomed neurons of the Drosophila CNS. Dev. Biol. 190: 206-213

37. Talbot WS, Swyryd EA and Hogness DS (1993) Drosophila tissue with different metamorphic responses to ecdysone express different ecdysone receptor forms. Cell 61: 101-111

38. Cho MK and Fahrbach SE (1995) Evidence for an endogenous neurocidin in the Manduca sexta ventral nerve cord. Arch. Insect Biochem. Physiol. 28: 273-289

39. Ready DF, Hanson TE and Benzer S (1976) Development of the Drosophila retina, a neurocrystalline lattice. Dev. Biol. 53: 217-240

40. Cagan RL and Ready DF (1989) The emergence of order in the Drosophila pupa retina. Dev. Biol. 136: 346-362

41. Reiter C, Schimansky T, Nie Z and Fischbach KF (1996) Reorganization of membrane contacts prior to apoptosis in the Drosophila retina: the role of the IrreC-rst protein. Development 122: 1931-1940

42. Cagan RL and Ready DF (1989) Notch is required for successive cell decisions in the developing Drosophila retina. Genes Dev. 3: 1099-1112

43. Muskavitch MA (1994) Delta-notch signaling and Drosophila cell fate choice. Developmental Biology 166: 415-430

44. Miller DT and Cagan RL (1998) Local induction of programmed cell death in the developing Drosophila retina. Development 125: 2327-2335

45. Freeman M (1994) Misexpression of the Drosophila argos gene, a secreted regulator of cell determination. Development 120: 2297-2304

46. Freeman M (1996) Reiterative use of the EGF receptor triggers differentiation of all cell types in the Drosophila eye. Cell 87: 651-660

47. Baker NE and Rubin GM (1989) Effect on eye development of dominant mutations in the Drosophila homologue of the EGF receptor. Nature 340: 150 153

48. Sawamoto K, Taguchi A, Hirota Y, Yumada C, Jin M and Okano H (1998) Argos induces programmed cell death in the developing Drosophila eye by inhibition of the Ras pathway. Cell Death Differ. 5: 262-270

49. Bergmann A, Agapite J, McCall Kand Steller H(1998) The Drosophila gene hid is a direct molecular target of Ras-dependent survival signaling [In Process Citation]. Cell 95: 331-341

50. Kurada $P$ and White K (1998) Ras promotes cell survival in Drosophila by downregulating hid expression [In Process Citation]. Cell 95: 319-329

51. Grether M, Abrams J, Agapite J, White Kand Steller H(1995) The head involution defective gene of Drosophila melanogasterfunctions in programmed cell death. Genes Dev. 9: 1674-1708

52. Ramos RGP, Igloi GL, Lichte B, Baumann U, Maier D, Schneider T, Brandstatter JH, Frohlich A, Fischbach KF. (1993) The irregular chaism C-roughest locus of Drosophila, which affects axonal projections and programmed cell death, encodes a novel immunoglobulin-like protein. Genes Dev. 7: 2533-2547

53. Schneider T, Reiter C, Eule E, Bader B, Lichte B, Nie Z, Schimansky T, Ramos RG, Fischbach KF. (1995) Restricted expression of the irreC-rst protein is required for normal axonal projections of columnar visual neurons. Neuron 15 : $259-271$

54. Gorski S, Brachmann CB, Tanenbaum S and Cagan RL (2000) Delta and Notch promote correct localization of IrreC-rst. Cell Death Differ. 7: 1011-1013

55. Wolff T and Ready DF (1991) Cell death in normal and rough eye mutants of Drosophila. Development 113: 825-839

56. Kooh PJ, Fehon RG and Muskavitch MA (1993) Implications of synamic patterns of Delta and Notch expression for cellular interactions during Drosophila development. Development, 117: 493-507

57. Bonini N, Leiserson W and Benzer S (1990) A mutation in compound eye development in Drosophilathat results in cell death rather than differentiation. J. Cell. Biochem. 14E: 502

58. Bonini NM and Fortini ME (1999) Surviving Drosophila eye development: integrating cell death with differentiation during the formation of a neural structure. BioEssays 21: $991-1003$ 
59. Tanenbaum S, Gorski S, Rusconi J and Cagan RL (2000) A screen for dominan modifiers of the irreC-rst cell death phenotype in the developing Drosophila retina. Genetics, 156: 205-217

60. Adachi-Yamada T, Gotoh T, Sugimura I, Tateno M, Nishida Y, Onuku T, Date H. (1999) De novo synthesis of sphingolipid is required for cell survival by downregulating c-JUN N-terminal kinase in Drosophila imaginal discs. Mol. Cell. Biol. 19: $7276-7286$

61. Leppa S and Bohmann D (1999) Diverse functions of JNK signaling and c-Jun in stress response and apoptosis. Oncogene 18: 6158-6162
62. Takatsu Y, Nakamura M, Stapleton M, Danos MC, Matsumoto K, O'Connor M, Shibuya H, Ueno N. (2000) TAK1 participates in c-Jun N-terminal kinase signaling during Drosophila development. Mol. Cell. Biol. 20: 3015-3026

63. Oeda E, Oka Y, Miyazono K and Kawabata M (1998) Interaction of Drosophila inhibitors of apoptosis with thick veins, a type I serine/threonine receptor for decapentaplegic. J. Biol. Chem. 273: 9353-9356

64. Namba R, Pazdera TM, Cerrone RL and Minden JS (1997)Drosophila embryonic repair: how embryos respond to bicoid dosage alteration. Development 124 : $1393-1403$ 